\title{
A coordinated control strategy based on Photovoltaic-hybrid energy storage system
}

\author{
Xiaobin Wang ${ }^{1, a^{*}}$, Hairong Zou ${ }^{2, b}$ \\ ${ }^{1}$ Department of Electrical Engineering, Shanghai Dianji University, Shanghai, 200120, China \\ ${ }^{2}$ Department of Electrical Engineering, Shanghai Dianji University, Shanghai, 200120, China
}

\begin{abstract}
Photovoltaic power generation is changing day by day, but its system has drawbacks. To compensate for the shortcomings of PV power generation system, the micro-grid system of PV power generation with HES is constructed by using the complementary characteristics of battery and supercapacitor. On the basis of this, we introduce a fuzzy control to realize the fast response of hybrid energy system. A micro-grid model is built based on Matlab/Simulink to verify the feasibility and rationality of the proposed control strategy.
\end{abstract}

\section{Introduction}

With the increasing environmental pollution problems and fossil energy consumption, people have to pay attention to the research and development of renewable energy sources. Although the advantages of renewable energy are outstanding, its inherent uncertainty and volatility have caused considerable headaches for researchers. The volatility of new energy sources can affect the quality of power supply and the stability of large power grids. To ensure the stable output of the power system, the role of energy storage devices comes to the fore. The research of PV-energy storage system is not too deep, so the research of PV-hybrid energy storage based micro-grid system is especially important, and the study of its operation characteristics and its control strategy has certain theoretical reference value and practical value.

In order to ensure the stable and reliable operation of the micro-grid system, the coordinated control strategy of the hybrid energy storage system is the key of research. In order to deeply study and solve the problem of control strategy of hybrid energy storage system, one literature regarded the vehicle system as a micro-grid system, with fuel cell power system as the main power source and battery as the auxiliary power source, and the main role of the battery is to absorb the fluctuating power. The literature [2] studied the energy storage technology of micro-grid containing battery, but due to the simple structure of the battery in it, the storage system is single, which leads to the complicated control of charging and discharging. The literature [3-5] proposed new methods and ideas for the topology of hybrid energy storage system, which makes the PV power generation unit work in the most ideal state by changing the position and connection of the converter in the system.

\section{Micro-grid system modeling}

\subsection{Photovoltaic cell model}

Photovoltaic power generation is the photovoltaic effect, the battery in the sunlight irradiation, through photoelectric conversion, the solar energy into electrical energy a way of power generation, its working principle in simple terms, people say photovoltaic effect. According to its principle, the equivalent circuit of a single photovoltaic cell is shown in the figure. The equivalent circuit is mostly the state of the ideal environment, in practice, the capacity of individual cells is very small and the voltage is relatively low, usually they are formed into photovoltaic arrays, that is, by connecting them in series and parallel.

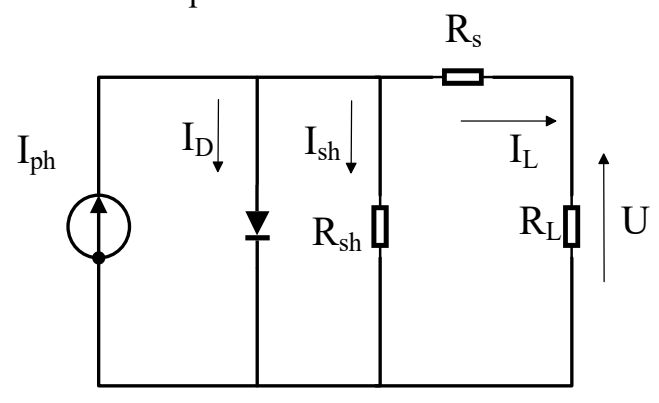

Figure 1. PV cell equivalent circuit

\footnotetext{
*Xiaobin Wang: ${ }^{\text {a } 849200653 @ q q . c o m}$

bzouhr@sdju.edu.cn
} 


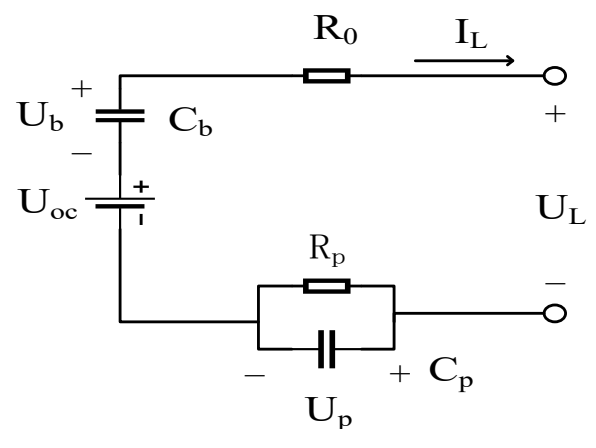

Figure 2. Equivalent circuit model of lithium battery

From Kirchhoff's current law, it follows that:

$$
I_{L}=I_{p h}-I_{s h}-I_{D}
$$

The relationship between the $P-N$ junction current and voltage is shown in the following equation.

$$
I_{D}=I_{0}\left(e^{q\left(U+I_{L} R_{S}\right)} / A K T-1\right)
$$

From Kirchhoff's voltage law, it follows that:

$$
\begin{gathered}
I_{S h}=\frac{U+I_{L} R_{S}}{R_{S h}} \\
I_{L}=I_{p h}-I_{0}\left(\left.e^{q\left(U+I_{L} R_{S}\right)}\right|_{A K T}-1\right)-\frac{\mathrm{U}+\mathrm{I}_{\mathrm{L}} \mathrm{R}_{S}}{\mathrm{R}_{\mathrm{Sh}}}
\end{gathered}
$$

Table1.Meaning of parameters

\begin{tabular}{|c|l|}
\hline Parameters Name & \multicolumn{1}{|c|}{ Meaning of parameters } \\
\hline$I_{L}$ & $\begin{array}{l}\text { Photovoltaic cell output } \\
\text { current }\end{array}$ \\
\hline$U$ & $\begin{array}{l}\text { Photovoltaic cell output } \\
\text { voltage }\end{array}$ \\
\hline$I_{p h}$ & $\begin{array}{l}\text { Photovoltaic cell photo- } \\
\text { generated current source } \\
\text { current }\end{array}$ \\
\hline$I_{0}$ & $\begin{array}{l}\text { Diode reverse saturation } \\
\text { current }\end{array}$ \\
\hline$q$ & Electron charge value \\
\hline$R_{s}$ & $\begin{array}{l}\text { Photovoltaic cell series } \\
\text { resistance }\end{array}$ \\
\hline$R_{s h}$ & $\begin{array}{l}\text { Photovoltaic cell parallel } \\
\text { resistance }\end{array}$ \\
\hline$K$ & Boltzmann constant \\
\hline$A$ & Diode ideal factor \\
\hline$T$ & $\begin{array}{l}\text { Photovoltaic cell operating } \\
\text { absolute temperature value } \\
\text { (K) }\end{array}$ \\
\hline
\end{tabular}

Equation (4) is a principle expression based on the equivalent circuit of $P V$ cell, which has been widely used in the theoretical study of $P V$ power generation, but many parameters in the expression are very sensitive to environmental changes and are difficult to adjust to an accurate value. In order to reduce the difficulty of model construction, the above model is simplified in engineering, and the simplified PV cell engineering model is shown in the figure below.

$$
\begin{aligned}
& I=I_{s c}\left\{1-C_{1}\left[e^{\frac{U}{C_{2} U_{o c}}}-1\right]\right\} \\
& C_{1}=\left(1-\frac{I_{m}}{I_{s c}}\right) e^{\left(-\frac{U_{m}}{C_{2} U_{o c}}\right)} \\
& C_{2}=\left(\frac{U_{m}}{U_{o c}}-1\right) / \ln \left(1-\frac{I_{m}}{I_{s c}}\right)
\end{aligned}
$$

Table2.Meaning of parameters

\begin{tabular}{|c|l|}
\hline$I_{s c}$ & Short-circuit current \\
\hline$U_{o c}$ & Open-circuit voltage \\
\hline$I_{m}$ & $\begin{array}{l}\text { Photovoltaic maximum } \\
\text { power point current }\end{array}$ \\
\hline$U_{m}$ & $\begin{array}{l}\text { Photovoltaic maximum } \\
\text { power point voltage }\end{array}$ \\
\hline
\end{tabular}

From equation (7), the voltagemetric characteristic curves of $P V$ cell output ( $P-V$ curve and $I-V$ curve) can be obtained by the four parameters of PV cell $I_{s c}, U_{o c}, I_{m}$ and $U_{m}$.

\subsection{Battery model}

By comparing the performance of different types of batteries, the results show that the performance of lithiumion batteries is more outstanding, so lithium-ion batteries are used. The common equivalent circuit models for lithium-ion batteries are the Rint model, in addition to the first-order resistance-capacitance model and the $P N G V$ equivalent circuit model. the $P N G V$ equivalent circuit model has a simple structure, consisting of resistance and capacitance, and is used to describe the operating characteristics of the battery. The physical meaning of this model is clear and the model parameters are relatively easy to determine. Moreover, its accuracy is higher than that of the simple first-order battery model, and it is the relatively most accurate equivalent circuit model for describing lithium batteries. The structure of the model circuit is shown in the following figure.

Table3.Meaning of parameters

\begin{tabular}{|l|l|}
\hline$U_{o c}$ & Open circuit voltage $(\mathrm{V})$ \\
\hline$R_{0}$ & Ohmic resistance $(\Omega)$ \\
\hline$R_{p}$ & Polarization resistance $(\Omega)$ \\
\hline$C_{p}$ & Polarization capacitance $(\mathrm{F})$ \\
\hline$I_{L}$ & Load current $(\mathrm{A})$ \\
\hline$U_{L}$ & Terminal voltage $(\mathrm{V})$ \\
\hline
\end{tabular}

From Kirchhoff's law, it follows that:

$$
\begin{aligned}
U_{o c} & =U_{L}+\frac{1}{c_{b}}\left(\int I_{L} d t\right)+R_{p} I_{p}+R_{0} I_{L} \\
U_{L, i} & =U_{o c}-\frac{1}{c_{b}} \times\left(\sum I_{L} \Delta t\right)_{i}-I_{L, i} \times R_{0}-I_{p, i} \times R_{p}(9)
\end{aligned}
$$

The relationship between the terminal current and the polarization current is given by:

$$
\begin{gathered}
\frac{d I_{p}}{d t}=\frac{\left(I_{L}-I_{P}\right)}{\tau} \\
I_{P}=I_{L}-\tau \times \frac{d I_{P}}{d t}
\end{gathered}
$$

\subsection{Super-capacitor model}

Super-capacitor energy storage is physical energy storage, unlike battery energy storage, there is no chemical reaction, so super-capacitor has high charging and discharging efficiency and fast charging and discharging speed. The most remarkable feature is long cycle life and 
high power density, which can respond to high frequency fluctuation of power.

There are many equivalent models of supercapacitor, but the $R C$ equivalent model of super-capacitoris is simple in structure and easy to obtain parameters, so it is widely used in simulation. In its equivalent model as shown in Figure3. a)

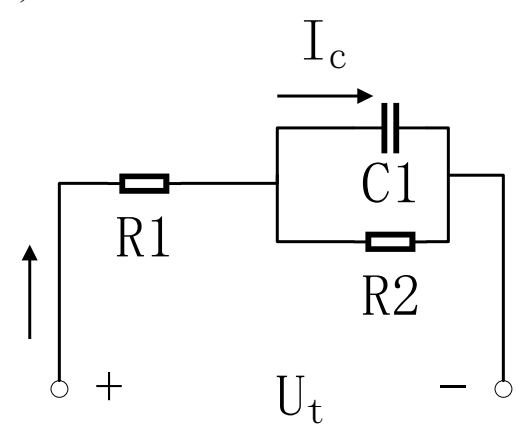

Figure 3.a) Classical model

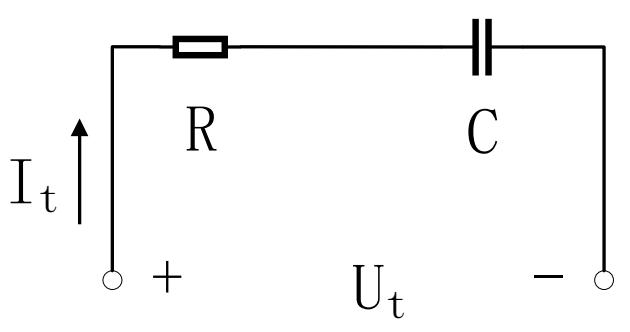

Figure 3.b) Simplified model

In order to simulate the super-capacitor leakage and better express the device characteristics, the researchers added a resistor $R 2$, which is connected in parallel with the capacitor, whose resistance value is generally large, and the current flowing through it is called leakage current, whose value is very small, generally only microampere, so it can be neglected in the calculation and simulation to obtain a simplified model of the super-capacitor, as shown in Figure3. b) below.

The relevant mathematical expressions are shown

$$
\begin{aligned}
& \mathrm{P}=U_{t} I_{t}=\left(U_{0}-I_{t} R\right) I_{t} \\
& \mathrm{C}=\frac{I_{t} d t}{d u} \\
& \mathrm{E}=\frac{1}{2} C U_{\text {max }}^{2}-\frac{1}{2} C U_{\text {min }}^{2} \\
& \eta=\frac{E_{d}}{E_{c}}=\frac{\int_{0}^{t} I_{d} \times u(t) d t}{\int_{0}^{t} I_{c} \times u(t) d t} \\
& \eta_{c}=\frac{\frac{1}{2} C\left(U_{c \max }^{2}-U_{c \min }^{2}\right)}{\int_{0}^{t} I_{c} \times u(t) d t} \\
& \eta_{d}=\frac{\int_{0}^{t} I_{d} \times u(t) d t}{\frac{1}{2} C\left(U_{d \max }^{2}-U_{d \min }^{2}\right)}
\end{aligned}
$$

$U_{0}$ is the initial voltage and $U_{t}$ is the terminal voltage; $U_{\max }$ and $U_{\min }$ are the maximum and minimum voltages allowed for the normal operation of super-capacitor; $E$ is the energy value of super-capacitor output; $I_{c}$ and $I_{d}$ are the charging and discharging currents; $\eta$ is the efficiency of super-capacitor; $E_{c}$ and $E_{d}$ are the charging and discharging energy; $\eta_{c}, \eta_{d}$ are the charging and discharging efficiency; $U_{c \max }$ and $U_{c m i n}$ are the charging initial voltage and cutoff voltage respectively; $U_{d \max }$ and
$U_{d \min }$ are the discharging initial voltage and cutoff voltage respectively.

\section{Selection of hybrid energy storage topology}

When the battery and super-capacitor are applied independently, they will have certain deficiencies because of their own characteristics and cannot meet the needs of the load and system well. Combined with the analysis of the characteristics of both, the two characteristics complement each other and can work well. The battery has high energy density and low power density, which is relatively reliable, but has a long response time to cope with sudden changes in power and is suitable for continuous discharge for a period of time; super-capacitor has high power density, long cycle life and fast response due to its own characteristics, but cannot be continuously discharged for a long time. The hybrid energy storage system in this paper consists of lithium battery and supercapacitor, and the advantages and disadvantages of their topologies are analyzed. The battery and super-capacitor are connected to the $D C$ bus through their respective $D C / D C$ converters, and the two $D C / D C$ converters are connected to the $\mathrm{AC}$ bus through a $D C / A C$ converter to ensure the stability of the bus power. In this structure, the super-capacitor and battery can be precisely controlled to avoid overcharging and over-discharging of the storage medium and prolong the service life. In addition, the hybrid energy storage system of this structure is simple and convenient to control, which can realize flexible energy management.

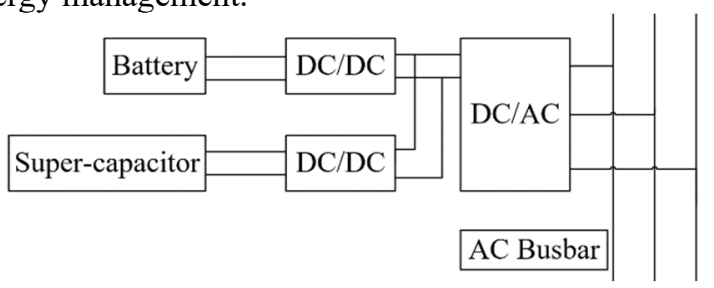

Figure 4. Dual DC/DC converter parallel structure

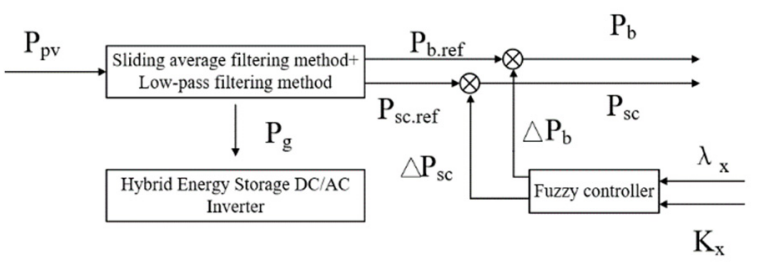

Figure 5. Control strategy structure diagram

\section{Hybrid energy storage system control strategy}

Hybrid energy storage systems combine batteries and super-capacitors. One of the reasons that affect the service life of the battery is the overcharge or overdischarge state of it. In order to avoid the battery to be in the deep charge and discharge state for a long time, the charge state SOC needs to be controlled and managed. Similarly, the overcharge and overdischarge of super-capacitor will also 
have a bad effect on its service life. Although the cycle life of super-capacitor is long, the cycle overcharge and overdischarge will not guarantee the long working time. Therefore, the battery and super-capacitor are well combined together to give full play to their respective advantages and improve the energy utilization of $P V$ power generation system.

The control method of the hybrid energy storage system is to obtain the grid-connected power and the reference power of the hybrid energy storage system by a sliding average filtering method. The reference power of the obtained hybrid energy storage system is decomposed by the low-pass filtering method, and the high-frequency component and low-frequency component are allocated to the super-capacitor and the battery, respectively. The fuzzy control method is used to regulate the SOC charging state of the energy storage to achieve the optimal energy distribution.

The control strategy structure of the system is shown in Fig5. The photovoltaic output power $P_{p v}$ is first applied to the sliding average filtering method to obtain the gridconnected power $P_{g}$ output to the grid. The hybrid energy storage reference power is decomposed by low-pass filtering method to get the low-frequency component and high-frequency component, respectively, to determine the reference power of lithium battery $P_{b . r e f}$ and the reference power of super-capacitor $P_{s c . r e f}$. In order to prevent the energy storage device from overcharging and overdischarging for a long time, increase the service life of the energy storage device, and ensure the $S O C$ charging state of the energy storage device is within a reasonable range, the fuzzy control is introduced to adjust the charging state. The charging state of the energy storage device and the reference power are used as the input of the fuzzy control to get the corrected power $\Delta P_{x}$ of the energy storage device, $\mathrm{x}$ is $\mathrm{b}$ for the lithium battery and $\mathrm{x}$ is sc for the super-capacitor. Finally, the reference power and the modified power of the energy storage device are added to obtain the adjusted charging and discharging power of the energy storage device.

\subsection{The fuzzy control}

The deep charging and discharging of energy storage devices is an important cause of their service life. To avoid overcharging and overdischarging of energy storage devices requires control management of the charge state SOC of energy storage devices. In this paper, we choose fuzzy control to regulate the charge state of the energy storage device. When the charge state is large, if the energy storage device is ready to charge, the charging power of the energy storage should be reduced, and if the energy storage is ready to discharge, the current power should be kept unchanged; when the charge state is small, if the energy storage is ready to charge, the original power should be maintained, and if the energy storage is ready to discharge, the discharge power of the energy storage should be reduced; when the charge state is within a reasonable range, the energy storage should be charged and discharged normally as instructed.
The charge state and reference power of the energy storage device are used as the input of the fuzzy controller, and the fuzzy input is normalized. The energy storage charge state subordinate degree function is as follows.

$$
\begin{aligned}
\lambda_{x} & =\frac{S O C_{x}-S O C_{x . r e f}}{S O C_{x . r e f}} \\
K_{x} & = \begin{cases}-\frac{1}{P_{x . \min }} \max \left[P_{x . \min },\right. & \left.P_{x . r e f}(t)\right] P_{x . r e f}(t) \leq 0 \\
\frac{1}{P_{x \text { max }}} \min \left[P_{x . \max },\right. & \left.P_{x . r e f}(t)\right] \quad P_{x . r e f}(t)>0\end{cases}
\end{aligned}
$$

Where $P_{x, \text { max }}, P_{x, \min }$ are the upper and lower limits of the output power of the energy storage device respectively

After normalization, $\lambda_{x}$ and $K_{x}$ are inputs to the fuzzy controller as input quantities, and the fuzzy domain is continuous domain $[-1,1]$. the fuzzy set of $\lambda_{x}$ is $\{N B$ (very low), $Z O$ (moderate), $P B$ (very high) $\}$. the fuzzy set of $K_{x}$ is $\{N B$ (negative large), $N M$ (negative medium), $Z O$ (zero), $P M$ (positive medium), $P B$ (positive large) $\}$. the fuzzy set of $K_{x}$ is $\{N B$ (negative large), $N M$ (negative medium), $Z O$ (zero), $P M$ (positive medium), $P B$ (positive large) $\}$. The output quantity is the power correction coefficient $\triangle$ $Y_{x}$ with discrete theoretical domain $[-1,-0.6,0,0.6,1]$ and fuzzy set $\{N B$ (negative large), $N M$ (negative medium), $Z O$ (zero), $P M($ positive medium), $P B$ (positive large)\}. The affiliation functions of the input quantities are shown below.

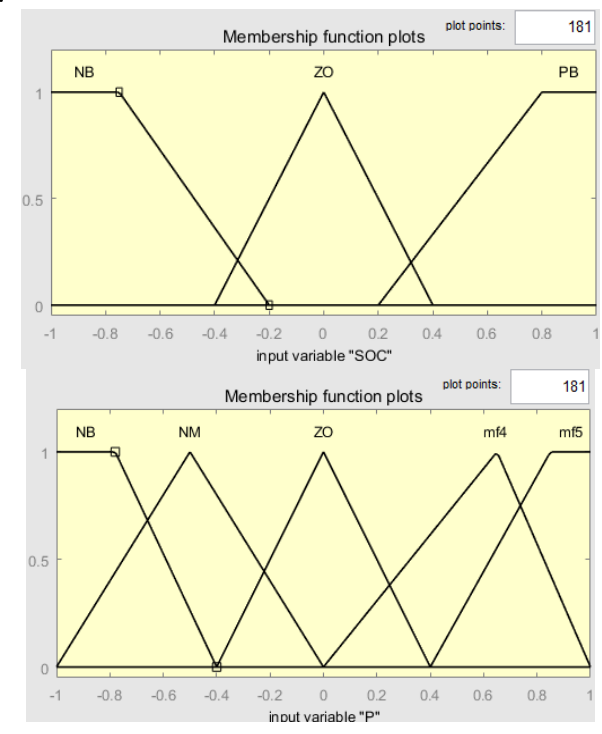

Figure 6. The affiliation function of the input quantity

The fuzzy control rules are shown in the table below.

Table 4. Fuzzy rule

\begin{tabular}{|c|c|c|c|c|c|}
\hline & \multicolumn{5}{|c|}{$K_{x}$} \\
$\lambda_{x}$ & $N B$ & $N M$ & $Z O$ & $P M$ & $P B$ \\
& & & $Z O$ & $N M$ & $N B$ \\
\hline$Z B$ & $Z O$ & $Z O$ & $Z O$ & & \\
\hline$Z O$ & $Z O$ & $Z O$ & $Z O$ & $Z O$ & $Z O$ \\
\hline$P B$ & $P B$ & $P M$ & $Z O$ & $Z O$ & $Z O$ \\
& & & & & \\
\hline
\end{tabular}




\section{Simulation verification}

In order to verify the effectiveness of the above control strategy, the micro-grid simulation model is built in Matlab/Simulink environment according to the topology shown in Figure 4, with some parameters set as follows: the target voltage of DC side is $700 \mathrm{~V}$, the target voltage of $\mathrm{AC}$ measurement is $380 \mathrm{~V}$, the rated voltage of $\mathrm{Li}$-ion battery pack is set to $200 \mathrm{~V}$, and the capacity is set to $100 \mathrm{Ah}$; the rated voltage of super The rated voltage of the super capacitor is set to $300 \mathrm{~V}$ and the capacitance value is set to $5 \mathrm{~F}$.

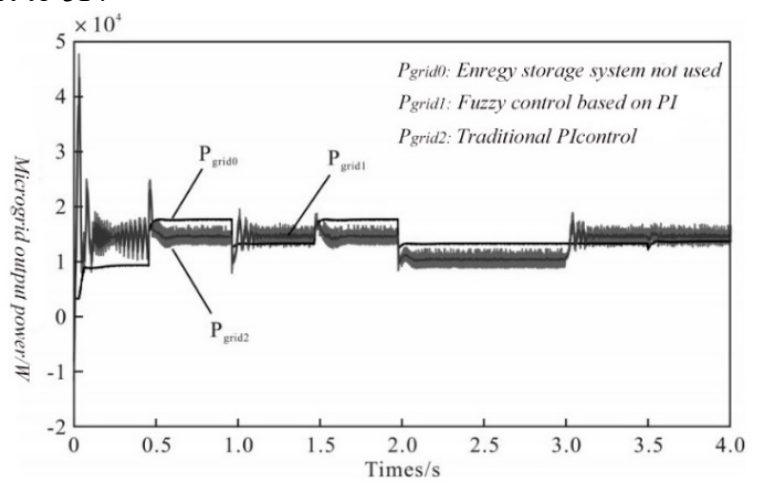

Figure 7. Micro-grid leveling power comparison

As shown in the figure, the power fluctuation curve of the PV cell is $P_{\text {grido }}$ in the state without using the energy storage device; after using the hybrid energy storage system, the $P_{\text {gridl }}$ curve in the figure is the power fluctuation curve after using the proposed fuzzy PI controller; as a comparison, $P_{\text {gridz }}$ is the power fluctuation curve when using the traditional PI controller. From the figure, it can be seen that the smoothing effect of using fuzzy controller is obviously better than that of using traditional PI controller, not only the response speed is faster, but also the curve is smoother compared.

\section{References}

1. Capacity and energy sharing platform with hybrid energy storage system: An example of hospitality industrySun L., Qiu J.,Han X., Yin X., DongZ.Y.(2020) AppliedEnergy, 280 , art. no. 115897

2. Sepehrzad R, Hassanzadeh M.E, Seifi A.R, et al. An efficient multilevel interconnect control algorithm in AC/DC micro-grids using hybrid energy storage system. 2021, 191, art.no.106869

3. Zhang Yuhan, Du Guiping, Lei Yanxiong. Energy management strategy with two degrees of freedom for hybrid energy storage systems in islanded DC microgrids. 2020, 13(14):3171-3179.

4. Cheng Long, Zhang Zehua, Zhang Fanghua. Secondary voltage controls of virtual-droop-controlled bidirectional DC/DC converters in hybrid energy storage system. 2020, 13(14):3018-3025.

5. Energy - Energy Storage; Researchers from National University Rio Cuarto Report Details of New Studies and Findings in the Area of Energy Storage (Energy
Management On Battery/ultracapacitor Hybrid Energy Storage System Based On Adjustable Bandwidth Filter and ...). 2020, :392-397.

6. Pablo A, Islam E.S, Bassam M, et al. Modeling, Simulationand Analysis of On-Board Hybrid Energy Storage Systems for Railway Applications. 2019, 12(11), art.no.2199 